Produto \& Produção, vol. 13 n. 1, p. 59-74, fev. 2012

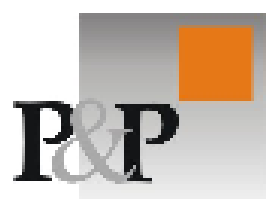

\title{
Identificação das propriedades da visão do produto no gerenciamento ágil de projetos de produtos manufaturados
}

Recebido em 21/11/2011. Aceito em 13/01/2012.

João Luis Guilherme Benassi

Departamento de Engenharia de Produção da Escola de Engenharia de São Carlos (EESC-USP)

jbenassi@sc.usp.br

Lucelindo Dias Ferreira Junior

Departamento de Engenharia de Produção da Escola de Engenharia de São Carlos (EESC-USP)

lucelindo@usp.br

\section{Daniel Capaldo Amaral}

Escola de Engenharia de São Carlos - Universidade de São Paulo amaral@sc.usp.br

O Gerenciamento Ágil de Projetos (APM) enfatiza e associa a visão do produto ao sucesso do projeto. Este argumento já era utilizado e verificado como verdadeiro por teóricos de outras áreas, como os de Gestão de Desenvolvimento do Produto. O APM inova na proposição de métodos para a geração de artefatos que caracterizariam a visão do produto antecipadamente, nas fases iniciais e mais nebulosas do projeto de produto. Um problema é que características positivamente associadas à visão do produto encontram-se dispersas na literatura, com terminologias confusas, dificultando a sua execução. Este trabalho apresenta uma revisão destas características que devem constituir uma visão do produto, empregando dois tipos de revisão bibliográfica: exploratória e sistemática. $\mathrm{O}$ resultado apresenta uma lista de características que tem como propósitos: ser uma referência para outros pesquisadores e, uma primeira iniciativa para medir a eficiência de métodos que apóiam a construção da visão do produto.

Palavras chave: Visão do Produto; Características da Visão do Produto; Gerenciamento Ágil de Projetos.

The Agile Project Management (APM) emphasizes and associates the product vision with de project success. This argument was already used and verified as true by theorists from other fields such as New Product Development. APM innovates in proposing methods for the generation of artifacts that characterize the product vision in the early and nebulous stages of the project. One problem is that characteristics positively associated with product vision are mixed and dispersed in the literature and 
so make it difficult to implement. This paper presents a review of these features that should be a product vision, using two types of literature review: exploratory and systematic. The result presents a list of features with two proposes: to be a reference for other researchers, and a first initiative to measure the effectiveness of methods that support the construction of the product vision.

Keywords: Product Vision; Product Vision Characteristics; Agile Project Management.

\section{INTRODUÇÃO}

O Gerenciamento Ágil de Projetos (APM) surge atualmente como uma alternativa para situações de projetos caracterizadas por alto grau de complexidade e incerteza, isto é, cujas metas e soluções estão pouco definidas no início do projeto (FERNANDEZ; FERNANDEZ, 2009). Essa abordagem caracterizada pelo emprego de ciclos curtos de desenvolvimento (iterações) e calcada em princípios como simplicidade e auto-gestão apresenta uma nova perspectiva para a gestão de projetos de produtos inovadores. Entre seus diferenciais está o conceito de visão do produto no lugar da Declaração de Escopo do Projeto, como pode ser visto em exemplos de Highsmith (2004) e Pichler (2010).

O conceito de visão em si, entretanto, não é novo. Foi identificado e tratado pelos teóricos de desenvolvimento de produto (COLLINS; PORRAS, 1996; BROWN; EISENHARDT, 1995) e nas medições empíricas recentes, tem se mostrado um dos componentes principais do sucesso, impactando, especialmente, na dimensão inovação (LYNN; AKGÜN, 2001; REID; BRENTANI, 2010).

Os autores do APM trazem algumas contribuições. A primeira é o enfoque em métodos que são utilizados para apoiar a construção da visão do produto e seus artefatos gerados, de forma a torná-la exeqüível, enquanto as literaturas de outras áreas sobre o tema tratam-na em um nível mais abstrato e teórico. A segunda contribuição refere-se à sugestão do uso desses métodos e artefatos no início do projeto, substituindo a Declaração de Escopo, diferindo das teorias tradicional de Gestão de Projetos (GP), e de Gestão de Desenvolvimento de Produtos (GDP) que indicam a utilização de tais métodos nas fases posteriores, sobretudo no projeto conceitual do produto.

Embora a relevância do uso da visão do produto e a utilização/presença de artefatos, elementos, e propriedades sejam ressaltadas por teóricos tanto da literatura de APM (HIGHSMITH, 2004; PICHLER, 2010), como da literatura de GDP (CLARK; FUJIMOTO, 1991; CHRISTENSON; WALKER, 2008), não se encontra atualmente um modelo conceitual de visão de produto ou, ainda, uma lista que possa unir e organizar todos estes termos em um só arcabouço. Essa deficiência dificulta a avaliação de métodos para apoiar a construção da visão do produto e a condução de estudos empíricos com a intenção de caracterizar a visão em ambientes reais de projeto.

O fenômeno construção da visão do produto possui várias dimensões: características objetivas, características subjetivas, fatores intervenientes, atividades e outras. O foco deste artigo é compilar uma das dimensões, as características subjetivas positivamente associadas, ora denominadas propriedades da visão do produto, dispersas na literatura. 
O trabalho visa obter, com base em revisão bibliográfica, uma síntese organizada das propriedades da visão do produto identificadas na literatura. $O$ resultado é a composição de uma lista de propriedades da visão do produto que possa ser aplicada no contexto do Gerenciamento Ágil de Projetos de produtos manufaturados. Percebe-se que há uma miríade de termos utilizados no âmbito do tema visão do produto, na teoria das áreas correlatas ao desenvolvimento de produtos. Esses termos são muitas vezes referidos nos textos como elementos, propriedades, ou artefatos. Aqui, utilizar-se-á o termo propriedades remetendo às características intrínsecas, de cunho qualitativo, relacionadas ao termo visão do produto. Adota-se o termo propriedades, pois, segundo o dicionário on-line OXFORD (2011), significa um atributo ou qualidade de algo, isto é, uma qualidade inerente (PRIBERAM, 2011).

Considera-se que as propriedades devem existir para que a visão do produto possa ser robusta e produza os benefícios citados pelos autores de APM e GDP. A determinação das propriedades é um passo importante para os teóricos da área, pois elas podem auxiliar a avaliação da visão do produto na prática, assim como o seu impacto no gerenciamento de projeto. Acredita-se que esse seja o primeiro passo para a definição dos contornos para a construção da visão de produtos manufaturados no contexto do Gerenciamento Ágil de Projetos.

\section{VISÃO DO PRODUTO}

A visão pode ser considerada um propósito compartilhado e um plano de ação que esclarece a missão, alinhamento estratégico e lista metas e prioridades do projeto que são consistentes com a capacidade interna da empresa e realidade do mercado (WHEELWRIGHT; CLARK, 1993). Assim, uma visão deve indicar um futuro a ser construído e não uma previsão (PARIKH; NEUBAUER, 1993). Nesse sentido, pode-se caracterizar a visão como uma forma de antecipação que instiga o preparo e o cuidado a áreas que são mais sensíveis a mudanças (SMITH, 2007).

Nota-se que o conceito visão tem sido empregado com freqüência em diversas áreas de estudo e níveis dentro de uma organização. Dentre estes, pode se citar estudos tratando da visão no nível organizacional (BROWN; EISENHARDT, 1995), empresarial (HAMEL; PRAHALAD, 1989; KOTTER, 1995) e, mercado (REID; BRENTANI, 2010). Além disso, este conceito é tratado também em áreas como: gestão de projetos (LYNN; AKGÜN, 2001; CHRISTENSON; WALKER, 2008) e, na Gestão do Desenvolvimento de Novos Produtos (WHEELWRIGHT; CLARK, 1993; O'CONNOR; VERYZER, 2001; TESSAROLO, 2007).

Um aspecto fortemente atrelado a esses trabalhos, porém pouco explorado, refere-se à visão do produto. Ao longo das duas últimas décadas o conceito seminal de visão do produto tem sido alimentado sutilmente por uma série de pesquisadores, muitas vezes usando terminologias diferenciadas. De acordo com Chen, Chang e Lin (2010), a visão do produto é uma declaração clara da direção e das metas dos mecanismos que habilitam a integração da companhia para rapidamente desenvolver novos produtos e assegurar que o progresso fique de acordo com 0 cronograma. Brown e Eisenhardt (1995) asseveram que a visão representa uma combinação entre competências e estratégias organizacionais e necessidades de mercado que tenciona criar um conceito de produto efetivo. Tessarolo (2007) capta essa definição e reforça dizendo que a visão do produto corresponde à definição de 
objetivos claros e de uma reconhecida estratégia para o Processo de Desenvolvimento de Produtos (PDP), que deve ser compartilhada entre todos os envolvidos. Nota-se que estas definições apresentam-se num nível alto de abstração, apenas tangenciando a intenção da visão do produto, sem indicar nitidamente quais as propriedades e como estruturar, de fato, uma visão nesse nível.

De modo a diminuir esta ambigüidade relacionada à visão a Figura 1 adaptada de Reid e Brentani (2010) apresenta os vários níveis de visão encontrados na literatura. Neste trabalho os autores demonstram que a visão de mercado e a competência em visão de mercado trazem vantagens como, por exemplo, o impacto positivo e precoce no desempenho do desenvolvimento de produtos inovadores. Apesar dos autores não tratarem do assunto visão do produto a Figura 1 adaptada do trabalho ajuda a compreender os níveis de visão da qual os autores de várias áreas se referem.

Além dos níveis de visão, a Figura 1 apresenta os níveis de foco e os mecanismos de ligação que conectam os níveis de visão. Em síntese, os níveis de foco podem ser definidos como o grau de abrangência da visão, enquanto os mecanismos de ligação, como as condições, elementos e competências que tornam os níveis de visão exequíveis ou operacionais.

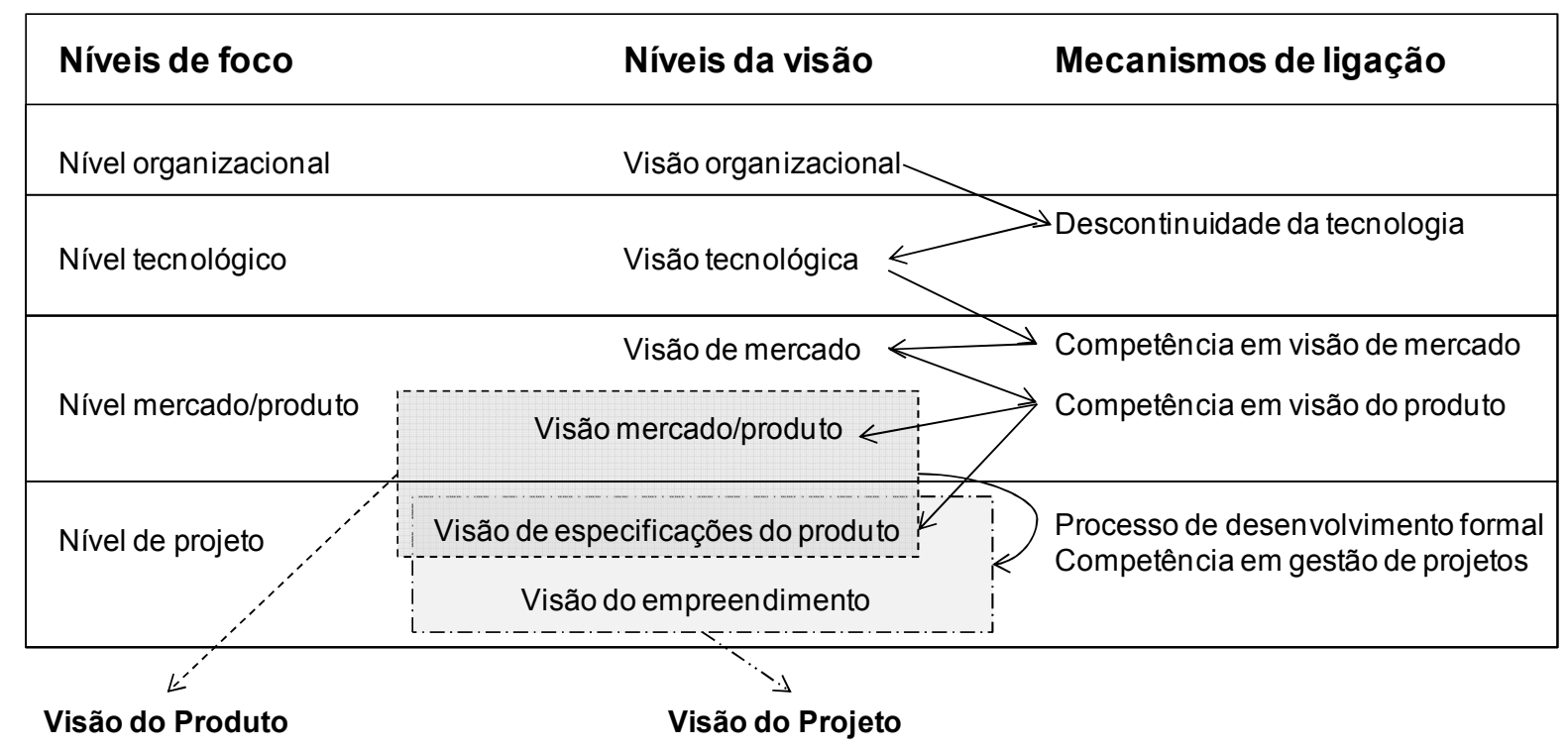

Figura 1 - Níveis de visão. Fonte: adaptado de Reid e Brentani (2010)

A Figura 1 também destaca a visão do produto e visão do projeto, superpostas, que possuem uma área comum denominada visão de especificações do produto. Cada uma delas é composta pela visão de mercado/produto e pela visão do empreendimento, requerendo um processo formal de desenvolvimento e competências (mecanismos de ligação) em áreas como, visão de mercado e produto, e gestão de projetos para se tornarem operacionalizáveis. Com relação à gestão de projetos nota-se que os autores começam a tratar mais o conceito de visão do produto. Em especial àqueles pertencentes ao APM. Dessa maneira, a próxima seção aborda esta questão. 


\section{VISÂO DO PRODUTO NO APM}

Autores pertencentes ao APM como Larman e Vodde (2010) e Highsmith (2004) consideram a visão do produto como uma descrição expandida do que o mesmo poderia se tornar servindo assim para limitar as suas características.

Schwaber e Beedle (2002) apud Pichler (2010) acrescentam que essa visão é uma descrição e/ou esboço que representa a essência do produto futuro, funcionando como uma meta global e guiando as pessoas envolvidas no projeto, isto é, consumidores, usuários, gerência, equipe de desenvolvedores e outros stakeholders.

Em linhas gerais observa-se na literatura de APM que os autores definem a visão do produto em relação à suas características, ou seja, artefatos, elementos, atores, e propriedades que, em conjunto, elucidariam a visão. Benassi e Amaral (2011) realizaram uma revisão bibliográfica que procurou levantar as características da visão do produto enumeradas por autores das áreas de gestão de desenvolvimento de produto e APM. Como resultado desta revisão notou-se que as definições encontradas eram abstratas e não forneciam elementos para a aplicação prática do conceito de visão do produto. A fim de tornar este conceito mais operacional Benassi e Amaral (2011) definiram visão do produto como uma descrição de alto nível, isto é, sucinta e preferencialmente na forma gráfica de um produto que ainda não existe e será entregue em um projeto. Os autores acrescentam que a visão pode conter dimensões como forma, função, possíveis estados, módulos e a interface entre eles, requisitos e metas.

Nota-se pelas definições encontradas na literatura que a visão do produto mencionada pelos diversos autores remete ao um termo bastante referido na literatura de gestão de desenvolvimento de produtos e denominado de "conceito". Segundo Koen et al (2002), conceito é uma forma bem definida, incluindo uma descrição escrita e visual, que abrange suas características principais e vantagens para o cliente juntamente com uma compreensão ampla da tecnologia necessária. De acordo com este contexto a visão do produto referida na teoria de Gerenciamento Ágil de Projetos poderia ser sobreposta ao termo conceito do produto, adicionando-lhe outras características objetivas, como é o caso da identificação preliminar de uma lista de materiais inicial, e, subjetivas, como a incorporação de qualidades tais como ser clara, motivadora, metafórica e etc.

Segundo Larman e Vodde (2010), o caráter parcialmente objetivo da visão do produto, no Gerenciamento Ágil de Projetos, visa proporcionar um alinhamento das idéias da equipe de projetos, mas, fundamentalmente, guiar o projeto para a entrega de características testáveis ao final de cada ciclo de desenvolvimento (iteração), facilitando a validação antecipada e gradativa das features do produto junto aos consumidores. Portanto conclui-se que a visão do produto e suas características não são uma ideia totalmente inovadora na literatura técnica e científica.

Os teóricos do APM inovam no sentido temporal do processo de desenvolvimento do produto, ou seja, eles propõem a utilização da visão do produto e de suas características em fases iniciais onde normalmente isso não era realizado durante a definição do escopo. Além disso, ela também substitui a tradicional Declaração de Escopo proposta pelo PMI (2008). O encorajamento para essa definição antecipada está relacionado à flexibilidade que a visão do produto possui no APM, podendo ser atualizada, ajustada ou corrigida ao término de cada iteração no PDP, com o auxílio dos consumidores (HIGHSMITH, 2004; PICHLER, 2010). 
A literatura revisada até o momento mostra a existência de trabalhos que definem o termo visão do produto. A próxima seção mostra também que existem vários trabalhos propondo métodos para se criar a visão. A identificação, e estudo, desses trabalhos é fundamental, já que tanto nos primeiros tipos de textos quanto nos segundos é possível a identificação das propriedades constitutivas da visão.

Optou-se pelo uso do termo propriedade, pois, segundo o dicionário on-line OXFORD (2011), este termo pode ser definido com um atributo ou qualidade de algo. Dessa maneira, considera-se no presente trabalho que as propriedades são os atributos ou qualidades da visão do produto que foram extraídas por meio da revisão bibliográfica realizada.

A próxima seção faz uma breve apresentação dos métodos para a criação da visão bem como suas principais fontes. Vale ressaltar que os métodos apresentados serviram também como base para o levantamento das propriedades da visão do produto.

\section{MÉTODOS PARA AUXILIAR A CONSTRUÇÃO DA VISÃO DO PRODUTO}

Devido à importância dada à visão do produto por autores das áreas de gerenciamento tradicional de projetos, APM e Desenvolvimento de Produtos, soluções contendo passos e ferramentas para auxiliar na sua descrição, já foram propostas. Tais soluções são rotuladas de ferramentas, métodos e, por vezes, práticas ágeis. Neste trabalho, será utilizado o termo método para denominá-las. De acordo como Engwall, Kling e Werr (2005), método é um procedimento ou meio articulado na forma de conhecimento específico, usado para resolver um problema específico.

Nas áreas de gerenciamento tradicional de projetos e APM, os trabalhos que se destacam na proposição de métodos são os de Highsmith (2004), Smith (2007) e Pichler (2010), que, em grande parte, se baseiam, ou trazem métodos igualmente referidos em outras áreas, como Marketing e PDP, a exemplo de Moore (1991) e Gray, Brown e Macanufo (2010). Os métodos identificados nessas literaturas foram: Elevator Statement Test (HIGHSMITH, 2004); Product Vision Box (HIGHSMITH, 2004; PICHLER, 2010); Feature Breakdown Structure (HIGHSMITH, 2004); Personas and Scenarios (SMITH, 2007; PICHLER, 2010); Trade Journal Review (PICHLER, 2010); Fused Deposition Modeling (HIGHSMITH, 2004); Case Uses (SMITH, 2007; PICHLER, 2010); User Stories (SMITH, 2007; PICHLER, 2010).

Por outro lado, os métodos encontrados nas áreas de Design de Produtos, Gestão do Desenvolvimento de Produtos e Design de Interfaces HumanoComputador, foram: Future Workshops (KENSING; MADSEN, 1991); Vision in Product Design (HEKKERT; VAN DIJK, 2001; LLOYD; HEKKERT; VAN DIJK, 2006); Visionary Concepts (LAITINEN et al, 2008; MERISTO et al. 2007; MERISTO et al, 2009); Vision-Oriented Innovative Product Process (LIN; LUH, 2009); Product Vision Management Method (BENASSI; AMARAL, 2011).

$\mathrm{Na}$ seção 5, será apresentado o método de pesquisa empregado para identificar os métodos supracitados e as propriedades da visão do produto. 


\section{MÉTODO E ETAPAS DE PESQUISA}

A pesquisa foi realizada em três etapas principais, como apresentado na Figura 2: revisão bibliográfica, análise teórica, e síntese de informações. A etapa de revisão bibliográfica foi desenvolvida em três momentos: revisão em textos referentes à teoria de APM; revisão em textos referentes à teoria de GDP e áreas correlatas; e, Revisão Bibliográfica Sistemática (RBS) em textos de áreas associadas ao desenvolvimento de produtos. Durante as revisões, foram identificados e revisados livros de leitura corrente, publicações periódicas e dissertações disponibilizadas por editoras, instituições educacionais ou alocados em bases de dados específicas para buscas por periódicos científicos (como, por exemplo, Science Direct, ISI Web of Knowledge, Emerald, Scopus, TEDE USP e etc.).

Inicialmente, revisou-se a literatura de Gerenciamento Ágil de Projetos com a intenção de delinear as características da visão do produto nessa área. Essa leitura teve caráter exploratório e consistiu na revisão de textos que abordavam a Gestão Ágil de Projetos de modo mais genérico, a exemplo de autores como Highsmith (2004), Chin (2004), De Carlo (2004), Boehm e Turner (2004), Benassi (2009) e Pichler (2010). Como as definições sobre visão do produto encontravam-se bastante esparsas, fez-se uma coleta e agrupamento das características referidas. Métodos sugeridos para a construção da visão do produto no APM foram igualmente reunidos, assim como exemplos de aplicação dos métodos, para facilitar a compreensão das características da visão. Sabendo-se que a teoria de APM está mais bem adaptada para o desenvolvimento de softwares, enquanto que o propósito do presente trabalho é a aplicação da visão do produto em produtos manufaturados, fez-se uma busca bibliográfica por outras teorias que abordassem o tema visão do produto. Autores como Brown e Eisenhardt (1995), Lynn e Akgün (2001), Tessarolo (2007), Reid e Brentani (2009) e Lange e Hehl-Lange (2010), em especial, fazem considerações interessantes sobre o tema em estudo, em áreas correlatas ao desenvolvimento de produtos, como por exemplo: Gestão do Desenvolvimento de Produtos, Marketing e Arquitetura e Urbanismo.

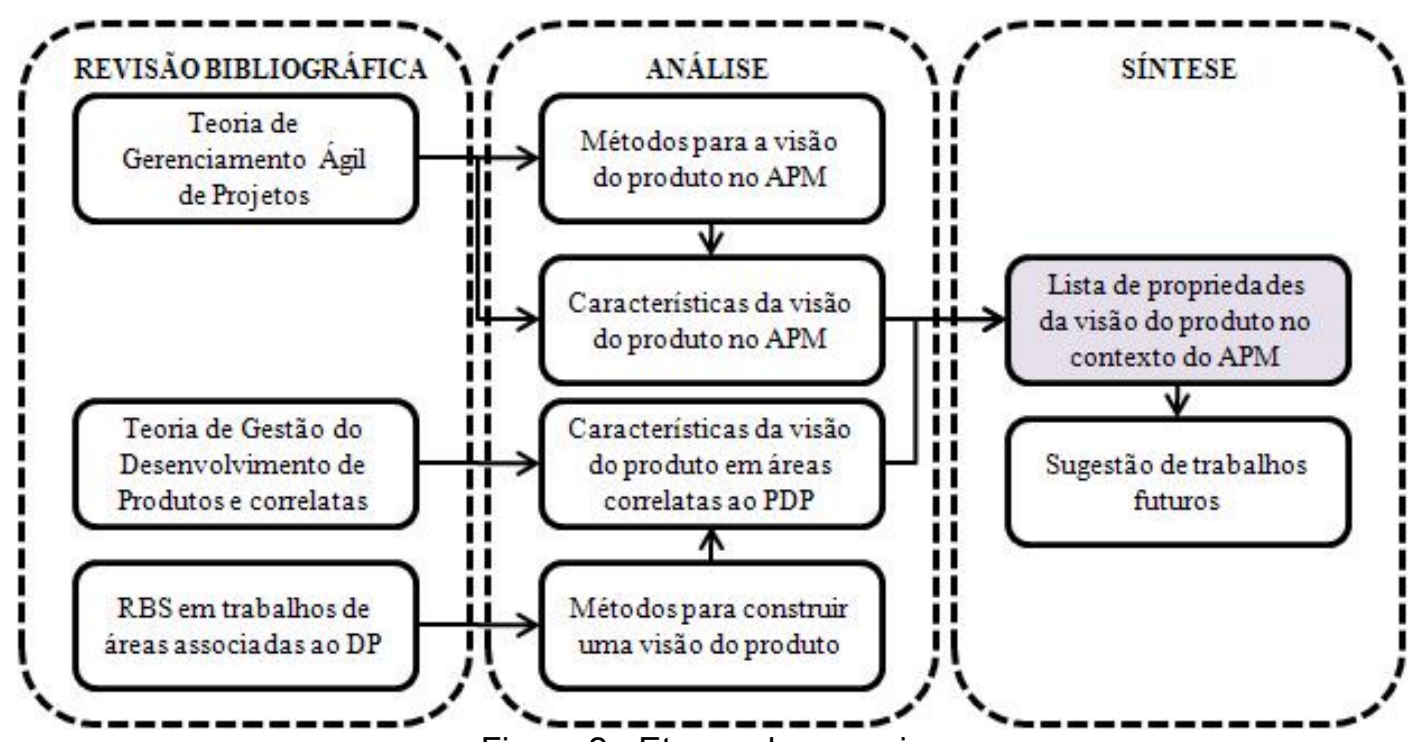

Figura 2 - Etapas da pesquisa 
Por fim, realizou-se uma Revisão Bibliográfica Sistemática (RBS) para encontrar métodos de apoio à construção da visão do produto, os quais foram apresentados na seção anterior. Essa atividade requisitou a definição de um protocolo de RBS no qual critérios para a inclusão/exclusão de trabalhos e métodos foram estipulados. Trabalhos como os de Kensing e Madsen (1991), Lloyd, Hekkert, e van Dijk (2006), Laitinen et al (2008), Lin e Luh (2009) e Benassi e Amaral (2011), apresentaram-se como as principais referências identificadas, de um total de 63 artigos pré-selecionados. Tais textos foram encontrados em revistas de áreas distintas, porém correlacionadas à área de desenvolvimento de produto, como Design de Produtos, Design de Interface Humano-Computador, Planejamento Urbano Comunitário, Gerenciamento de Projetos, por exemplo.

Foram selecionados os métodos que atendiam aos seguintes critérios: (a) ser declaradamente para a construção de algo similar a uma visão do produto; (b) apresentar as características básicas de uma visão do produto no APM, conforme sintetizado por Benassi e Amaral (2011), ou seja, (b1) contar com informações básicas de Marketing (elevator statement test) (MOORE, 1991 apud HIGHSMITH, 2004); (b2) descrever parcial, ou integralmente, o escopo do produto, isto é, evidenciando benefícios, forma, tecnologias, desempenho esperado e funcionalidades (ROZENFELD et al, 2006; CRAWFORD; DI BENEDETTO, 2006); (b3) envolver o consumidor no processo de construção da visão em qualquer um dos níveis de participação considerados por Kaulio (1998). Segundo essa classificação em níveis, o envolvimento do consumidor pode ser mínimo, como é o caso em que a equipe de projeto utiliza somente dados de mercado ou observação do consumidor, ou máximo, quando o consumidor torna-se parte da equipe de desenvolvedores, decidindo e influenciando diretamente no projeto do produto.

Como resultado da análise da literatura revisada, identificou-se referências teóricas aos atributos que uma visão do produto deveria possuir e, em adição, as características da visão do produto, através de análise dos métodos utilizados para apoiar a elaboração da visão do produto citados na literatura de APM e em áreas correlatas ao desenvolvimento de produtos. Essa análise culminou na constituição de um conjunto de características subjetivas, que serão denominadas aqui como propriedades, essenciais a uma visão do produto. Essas propriedades estavam apresentadas de forma dispersa na literatura e, normalmente, associadas a benefícios considerados intrínsecos a uma visão do produto, como será visto na seção 6.

\section{PROPRIEDADES IDENTIFICADAS PARA A VISÃO DO PRODUTO}

$\mathrm{Na}$ Figura 3 encontra-se a lista de propriedades para a visão do produto. $\mathrm{Na}$ seqüência há uma descrição do significado de cada propriedade e seus benefícios no Processo de Desenvolvimento de Produtos, conforme sugerido em estudos empíricos ou teóricos sobre o tema. 


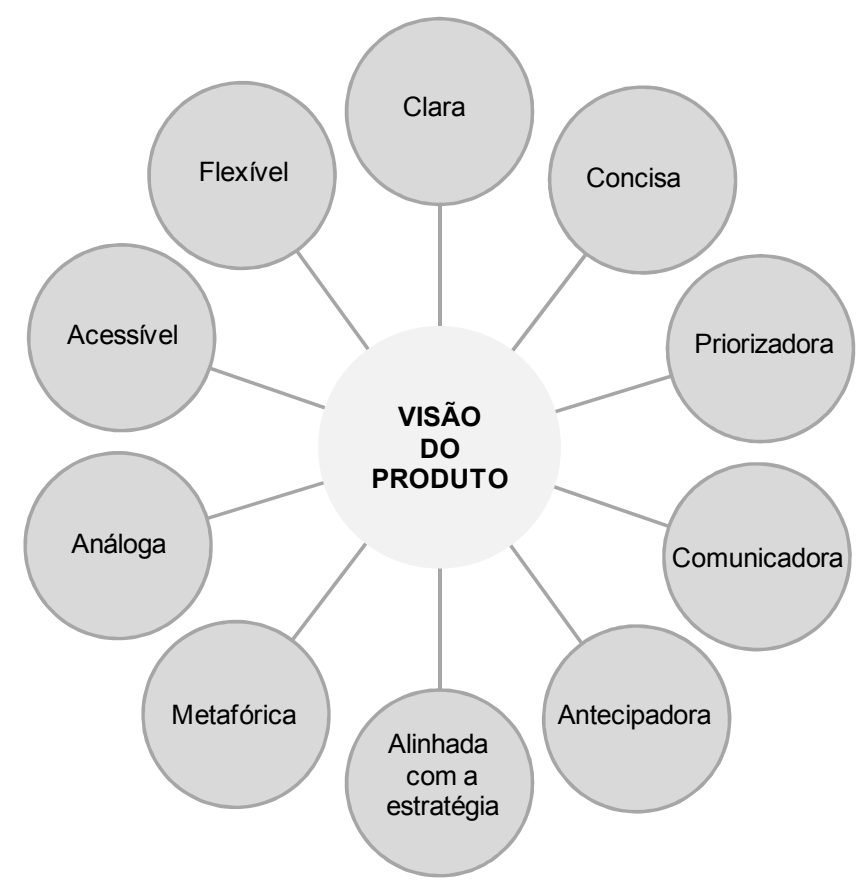

Figura 3 - Propriedades da visão do produto

Propriedades 1 e 2: Clara e Concisa. Dentre os benefícios atribuídos ao uso da visão do produto, muitos se relacionam à sua capacidade de comunicar e sintetizar, numa base comum, informações essenciais sobre o projeto do produto. Lynn e Akgün (2001), encontraram, em um estudo empírico, que uma definição clara da visão do produto, indicando características do produto, mercado alvo, preço-meta e etc., estava significantemente ligada ao sucesso de produtos contendo inovações radicais. Essas propriedades são constantemente associadas à visão do produto, sendo referidas por Highsmith (2004), Pichler (2009) e Lange e Hehl-Lange (2010). A clareza da visão do produto está, em parte, ligada à sua capacidade em representar visualmente o conhecimento tácito crítico sobre conceitos de produtos complexos e idéias, que facilita a tomada de decisões e influencia positivamente no resultado do projeto (YAP; NGWENYAMA; OSEI-BRYSON, 2003). Tessarolo (2007) acrescenta que uma visão do produto claramente delineada pode acelerar o tempo de desenvolvimento do produto.

Propriedade 3: Priorizadora. No Gerenciamento Ágil de Projetos a visão do produto é considerada a big picture, descrevendo de maneira clara a razão pela qual o projeto está sendo empreendido e as principais características que o produto deve ter ao final de todas as entregas (HIGHSMITH, 2004; LARMAN; VODDE, 2010). Nesse sentido, cabe à visão do produto apresentar as características primordiais no projeto do produto, já que há um espaço limitado para a representação da visão, devido à sua necessidade em ser concisa. Para Pichler (2010) essa priorização deve ser feita de modo que os elementos selecionados da visão do produto possam atender a uma maior quantidade de necessidades dos consumidores. Nesse sentido, é interessante verificar que a priorização pode ser útil para eliminar conflitos entre atributos desejáveis do produto (PICHLER, 2010).

Propriedade 4: Comunicadora. Lange e Hehl-Lange (2010) reforçam que a visão do produto não deve ser apresentada apenas por meio de uma declaração global ou composição de gráficos, mas através do uso de imagens claras que permitam uma compreensão, incluso, de pessoas leigas. Corroboram com essa 
ideia Pearson e Gorman (2010), que acreditam que a visão deve ser originada de um processo de trabalho em conjunto e deve ser de fácil compreensão permitindo ampla comunicação entre os envolvidos. O modelo de representação deve ser visualmente compreensível de forma a incentivar uma participação mais ativa e ampliar o número de integrantes. Para isso Lange e Hehl-Lange (2010) recomendam o uso de ferramentas para representação em 3D, embora considerem esse tipo de aplicação muito escasso em casos reais de projetos. Tessarolo (2007) identificou que a visão do produto ao comunicar eficientemente as informações relacionadas ao projeto, alinhando as perspectivas funcionais com as metas de desenvolvimento, proporcionava tanto integração interna quanto externa, participação direta de stakeholders nos estágios iniciais do PDP e diminuição no tempo de desenvolvimento do produto.

Propriedade 5: Antecipadora. De acordo com Säde (1999), a representação visual/física de conceitos nos estágios iniciais do PDP permite que haja um maior espaço para a geração, comunicação e teste de ideias, mesmo que através de modelos de baixa qualidade, provendo informações para a tomada de decisões no processo de design. Essa abordagem permite a ocorrência de mudanças quando o custo para a sua realização é menor (SMITH, 2007). Além disso, essa capacidade de antecipar permite uma orientação da equipe de projeto de produto, que é fundamental no planejamento, pois possibilita uma estimativa de custo e tempo de duração, além de concentrar os esforços da equipe à realização de um objetivo comum.

Propriedade 6: Alinhada com a estratégia. Segundo Laitinen et al (2008), a representação da visão do produto pode ocasionar um estreitamento das ligações entre a alta administração e o setor de pesquisa e desenvolvimento, fazendo com que cada grupo possa perceber melhor a perspectiva um do outro. Além de ajudar a empresa a comunicar a Visão e Missão para as outras pessoas da organização ao nível do produto.

Propriedades 7 e 8: Descreve em termos de Analogias e Metáforas. Kensing e Madsen (1991) e Amaral et al (2011), sugerem que a visão do produto deve ser desenvolvida utilizando metáforas e analogias, com a intenção de ampliar as perspectivas dos usuários e fomentar a produção criativa no desenvolvimento da visão. Para Nonaka (2001), o uso da metáfora é importante na construção da visão, pois é uma forma de tornar intuitiva, através de símbolos, a compreensão de algo por pessoas com níveis de entendimento distintos, sendo capaz de facilitar o comprometimento e a criatividade dos envolvidos. O autor considera que o processo de conversão do conhecimento tácito em explícito ocorre em termos ideais através da compreensão intuitiva de objetos e ideias contraditórias por meio de metáfora, seguida da análise dos aspectos contraditórios da metáfora por analogia e, por fim, pela compilação dos resultados em um modelo real, que terá como conteúdo o conhecimento explícito a ser difundido na organização. Embora esse modo de representação utilize modelos essencialmente distantes dos que serão desenvolvidos, eles provém informações para a tomada de decisões no processo de design antecipadamente (SÄDE, 1999), permitindo a representação visual do conhecimento tácito crítico sobre conceitos de produtos complexos e idéias (YAP; NGWENYAMA; OSEI-BRYSON, 2003).

Propriedade 9: Acessível. Lange e Hehl-Lange (2010) sugerem que deve existir uma manipulação e difusão das Visões coletivas em um espaço virtual de troca de informações pela internet, ampliando, assim, o envolvimento das partes que serão afetados. Haine (2009), em seu método SSNiF, apresenta uma forma de 
compartilhamento das visões do produto o que permitiria a inclusão de um grande número de participantes, tanto pela forma de acesso, que pode ser feita através da internet. Ademais, todos os envolvidos no projeto do produto estariam cientes das visões do produto criadas, podendo sugerir alterações e/ou validar em tempo real, em qualquer momento.

Propriedade 10: Flexível. No Gerenciamento Ágil de Projetos, a visão do produto é originada/criada antecipadamente, nas fases iniciais e mais nebulosas do projeto de produto. O encorajamento para essa definição antecipada está relacionado à flexibilidade que a visão do produto possui no APM, podendo ser atualizada, ajustada ou corrigida ao término de cada iteração do projeto de produto, com o auxílio dos consumidores (HIGHSMITH, 2004; PICHLER, 2009). Lynn e Akgün (2001) adicionam referindo que essa capacidade de depuração da visão está significantemente associada a projetos radicais, nos quais há elevado grau de desconhecimento sobre mercado e tecnologias no início do projeto. Nesses casos, a estabilidade pode não ser crítica e a visão pode ser implícita até fases mais posteriores no PDP (LYNN; AKGÜN, 2001). Thomke e Reinertsen (1998) declaram que essa abordagem permite o design flexível e a incorporação de informações mais precisas ao tempo em que se avança no projeto do produto. Apesar disso, o conceito core do projeto não deve ser perdido e a visão deve permanecer consistente com o propósito inicial durante o projeto (CHRISTENSON; WALKER, 2008).

\section{CONSIDERAÇÕES FINAIS}

A Figura 3, da seção anterior, apresenta as propriedades que devem compor a visão do produto, segundo os teóricos da bibliografia revisada. A revisão foi realizada em três momentos da pesquisa e contou com uma Revisão Bibliográfica Sistemática, na qual se identificou autores de áreas correlatas ao desenvolvimento de produtos que discerniam sobre o tema visão do produto propondo, incluso, métodos para a sua operacionalização, em termos de produtos manufaturados. A literatura escolhida e revisada foi identificada em livros, publicações periódicas e dissertações presentes em bases de dados reconhecidas no meio científico acadêmico, como Science Direct, Emerald, Web of Science, IEEE Xplore Digital Library, TEDE USP, e etc.

As propriedades levantadas, conforme encorajado pela literatura, devem estar presentes em sua totalidade para constituir uma visão do produto adequada no contexto do Gerenciamento Ágil de Projetos de produtos manufaturados. Relativamente a essa consideração, acredita-se que um estudo prático do impacto conjunto das propriedades deve ser conduzido, de modo a propiciar um entendimento mais realista do tema, embora, algumas das propriedades, isoladamente, tenham sido verificadas por autores supracitados. Esses autores, como é o caso, por exemplo, de Lynn e Akgün (2001) e Lange e Hehl-Lange (2010), apresentam indícios que a presença de algumas das propriedades, como "flexível", "comunicadora" e "acessível", aumenta as chances de sucesso do projeto. Por outro lado, propriedades como "priorizadora" e "antecipadora", embora citadas como indispensáveis e relacionadas a resultados positivos no projeto, por autores como Highsmith (2004) e Smith (2007), carecem de maior exploração empírica, sobretudo quanto às suas exequibilidades nas fases iniciais do projeto. De qualquer modo, é 
interessante notar que as propriedades possuem uma ligação forte entre si, e a difícil aplicação de uma propriedade como a "priorizadora" no início do projeto, pode ser atenuada com o uso da propriedade "flexível". Nesse sentido, a priorização de certos elementos do produto na visão, deve ser passível de correção, ao invés de funcionar como uma propriedade que irá enrijecer o desenvolvimento do produto. A despeito de tais considerações de cunho teórico, acredita-se que a identificação da relação entre o resultado do projeto e o conjunto de propriedades listadas é uma aposta interessante para trabalhos futuros.

Por fim, acredita-se que a lista proposta no artigo é um passo inicial na caracterização da visão do produto, de acordo com as necessidades do Gerenciamento Ágil de Projetos. Sendo, portanto, indispensável na posterior avaliação de métodos para apoiar a descrição da visão do produto; e, também, no consequente aprimoramento desses métodos. Espera-se que o conjunto de propriedades ora referidos, possa figurar como uma lista de referência das propriedades da visão do produto. Sugere-se, ainda, para trabalhos posteriores, um estudo aprofundado acerca das outras dimensões da visão do produto, tais como características objetivas, fatores intervenientes, atividades inerentes à construção da visão, entre outras, de forma a constituir um arcabouço teórico que possa delinear, de maneira bastante completa, o perfil da visão do produto no contexto do Gerenciamento Ágil de Projetos de produtos manufaturados.

\section{REFERÊNCIAS}

AMARAL, D. C.; CONFORTO, E. C.; BENASSI, J. L. G.; ARAUJO, C. Gerenciamento Ágil de Projetos: aplicação em produtos inovadores. São Paulo: Saraiva, 2011.

BENASSI, J. L. G. Avaliação de modelos e proposta de método para representação da visão do produto na gestão ágil de projetos. São Carlos: EESC-USP, 2009. 179 p. Dissertação (Mestrado em Engenharia de Produção) - Programa de PósGraduação em Engenharia de Produção, Escola de Engenharia de São Carlos, Universidade de São Paulo, São Carlos, 2009.

BENASSI, J. L. G.; AMARAL, D. C. Método para a descrição da visão do produto no contexto do gerenciamento ágil de projetos. Revista Produção, v. 21, n. 3, p. 392403, 2011.

BOEHM, B.; TURNER, R. Balancing agility and discipline: A guide for the perplexed. Boston: Pearson, 2004.

BROWN, S. L.; EISENHARDT, K. M. Product development: Past research, present finds, and future directions. Academy of Management Review, v. 20, n. 2, p. 343378, 1995.

CHEN, C. H.; CHANG, Y. Y.; LIN, M. J. J. The performance impact of post-M\&A interdepartmental integration: An empirical analysis. Industrial Marketing Management, v. 39, n. 7, p. 1150-1161, 2010. 
CHIN, G. Agile project management: How to succeed in the face of changing project requirements. New York: Amacon, 2004.

CHRISTENSON; D.; WALKER, D. H. T. Using vision as a critical success element in project management. International Journal of Managing, v. 1, n. 4, p. 611-622, 2008.

CLARK, K. B.; FUJIMOTO, T. Product development performance: Strategy, organization, and management in the world auto industry. Boston: Harvard Business School Press, 1991.

CLARK, K. B.; WHEELWRIGHT, S. C. Managing new product and process development: Text and cases. New York: Free Press, 1993.

COLLINS, J. C.; PORRAS, J. I. Building your company's vision. Harvard Business Review, p. 65-77, set/out 1996.

CRAWFORD, C. M.; DI BENEDETTO, C. A. New products management. New York: McGraw-Hill, 2006.

DECARLO, D. Extreme project management: Using leadership, principles, and tools to deliver value in the face of volatility. San Francisco: Jossey-Bass, 2004.

ENGWALL, M.; KLING, R.; WERR, A. Models in action: how management models are interpreted in new product development. R\&D Management, v. 35, n.4, p. 427439, 2005.

FERNANDEZ, D. J.; FERNANDEZ, J. D. Agile Project management: Agilism versus traditional approaches. The Journal of Computer Information Systems, v. 49, n. 2, p. $10-17,2008 / 2009$.

GRAY, D.; BROWN, S.; MACANUFO, J. Gamestorming: A playbook for innovators, rulebrakers, and changemakers. Sebastopol: O’Reilly Media, 2010.

HAINE, P. Product vision book. Disponível em: <http://productvision.org/blog/>. Acesso em: 08 set. 2010.

HAMEL, G.; PRAHALAD, C. K. Strategic intent. Harvard Business Review, p. 63-76, may/june 1989.

HEKKERT, P.; VAN DIJK, M. Designing from context: Foundations and applications of the ViP approach. In: Lloyd, P. \& Christiaans, H. (ed.). Designing in context: Proceedings of design thinking research symposium 5. Amsterdam: Delft University Press, 2001.

HIGHSMITH, J. Agile project management: Creating innovative products. Boston: Addisson-Wesley, 2004.

KAULIO, M. A. Customer, consumer and user involvement in new product development: A framework and a review of selected methods. Total Quality Management, v. 9, n. 1, p. 141-149, 1998. 
KENSING, F.; MADSEN, K. H. Generating Visions: Future Workshops and Metaphorical Design. In: Greenbaum, J. \& Kyng, M. (ed.). Design at work: Cooperative design of computer systems. New Jersey: Lawrence Erlbaum Associates, 1991.

KOEN, P. A.; AJAMIAN, G. M.; BOYCE, S.; CLAMEN, A.; FISHER, E.; FOUNTOULAKIS, S.; JOHNSON, A.; PURI, P.; SEIBERT, R. Fuzzy front end: Effective methods, tools, and techniques. In: Belliveau, P.; Griffin, A.; Somermeyer, S. (eds.). The PDMA Toolbook for New Product Development. New York: Wiley, 2002.

KOTTER, J. P. Leading change: Why transformation efforts fail. Harvard Business Review, v. 73, p. 59-67, march/april 1995.

LAITINEN, J.; LEPPIMAKI, S.; MERISTO, T.; TUOHIMAA, H. Visionary concept: Combining scenario methodology with concept development. In: Wagner, C. G. (ed.). Seeing the future through new eyes. Washington: World Future Society, 2008.

LANGE, E.; HEHL-LANGE, S. Making visions for long-term landscape management. Futures, n. 42, p. 693-699, 2010.

LARMAN, C.; VODDE, B. Practices for scaling lean and agile development: Large, multisite, and offshore product development with large-scale Scrum. London: Addison-Wesley, 2010.

LIN, C.; LUH, D. A vision-oriented approach for innovative product design. Advanced Engineering Informatics, n. 23, p. 191-200, 2009.

LLOYD, P.; HEKKERT, P.; VAN DIJK, M. Vision in product design: The warm bath. Amsterdam. 2006. Disponível em: <http://bluehaired.com/corner/wpcontent/uploads/2008/10/vip-booklet.pdf >. Acesso em: 10 set. 2010.

LYNN, G. S.; AKGÜN, A. E. Project visioning: Its components and impact on new product success. The Journal of Product Innovation Management, v. 18, p. 374-387, 2001.

MERISTO, T.; KETTUNEN, J.; LEPPIMAKI, S.; LAITINEN, J. Competitive advantage through market-oriented innovation process: Applying the scenario approach to create radical innovations. In: XVIII ISPIM Annual Conference Innovation for Growth, 2007, Warsaw. Proceedings of XVIII ISPIM Annual Conference Innovation for Growth. Warsaw: ISPIM, 2007.

MERISTO, T.; LEPPIMAKI, S.; LAITINEN, J.; TUOHIMAA, H. Scenario Filter Model: The framework to generate scenarios and select visionary concepts for development. In: XX ISPIM Annual Conference Innovation for Growth, 2009, Vienna. Proceedings of XX ISPIM Annual Conference Innovation for Growth. Vienna: ISPIM, 2009.

MOORE, G. A. Crossing the chasm: Marketing and selling high-tech products to mainstream customers. New York: HarperBusiness, 1991. 
NONAKA, I. A empresa criadora de conhecimento. In: Harvard Business Review. Gestão do conhecimento. Rio de Janeiro: Campus, 2001.

O'CONNOR, G. C.; VERYZER, R. W. The nature of market visioning for technologybased radical innovation. The Journal of Product Innovation Management, v. 18, p. 231-246, 2001.

OXFORD DICTIONARIES. Disponível em: < http://oxforddictionaries.com/>. Acesso em: $12 / 11 / 2011$.

PARIKH, J.; NEUBAUER, F. Corporate Visioning. In: Hussey, D. E. (ed.). International Review of Strategic Management. Chichester: Wiley, 1993.

PEARSON, D. M.; GORMAN, J. T. Managing the landscapes of Australian Northern Territory for sustainability: Visions, issues and strategies for successful planning. Futures, v. 42, p. 711-722, 2010.

PICHLER, R. Agile product management with Scrum: Creating products that customers love. London: Addison-Wesley, 2010.

PRIBERAM. Dicionário Priberam da língua portuguesa. Disponível em: < http://www.priberam.pt>. Acesso em: 12/11/2011.

PROJECT MANAGEMENT INSTITUTE (PMI). Um Guia do Conhecimento em Gerenciamento de Projetos (Guia PMBOK®). 4 ed. Pennsylvania: 2008.

REID, S. E.; BRENTANI, U. Market vision and market visioning competence: Impact on early performance for radiacally new, high-tech products. Journal of Product Innovation Management, v. 27, p. 500-518, 2010.

ROZENFELD, H.; AMARAL, D. C.; FORCELLINI, F. A.; TOLEDO, J. C.; SILVA, S. L.; ALLIPRADINI, D. H.; SCALICE, R. K. Gestão de desenvolvimento de produtos: Uma referência para a melhoria do processo. São Paulo: Saraiva, 2006.

SÄDE, S. Representations of smart product concepts in user interface design. In: Green, W. S.; Jordan, P. W. (ed.). Human factors in product design: Current practice and future trends. New York: Taylor and Francis, 1999.

SMITH, P. G. Flexible product development: Building agility for changing markets. San Francisco: Jossey-Bass, 2007.

SCHWABER, K.; BEEDLE, M. Agile software development with Scrum. New Jersey: Prentice Hall, 2002.

TESSAROLO, P. Is integration enough for fast product development? An empirical investigation of the contextual effects of product vision. The Journal of Product Innovation Management, v. 24, p. 69-82, 2007. 
THOMKE, S.; REINERTSEN, D. Agile product development: Managing development flexibility in uncertain environments. California Management Review, v. 41, n. 1, p. 830, 1998.

YAP, A. Y.; NGWENYAMA, O.; OSEI-BRYSON, K. Leveraging knowledge representation, usage, and interpretation to help reengineer the product development life cycle: visual computing and the tacit dimensions of product development. Computers in Industry, v. 51, p. 89-110, 2003. 